\title{
Mechanical Engineering of Leg Joints of Anthropomorphic Robot
}

\author{
Nikita Pavluk ${ }^{1}$, Alexander Denisov ${ }^{1}$, Andrey Kodyakov ${ }^{2}$ and Andrey Ronzhin ${ }^{1,2}$ \\ ${ }^{1}$ SPIIRAS 39, 14 line, St. Petersburg, 199178, Russia \\ ${ }^{2}$ SUAl, 67, Bolshaya Morskaia str., St. Petersburg, 190000, Russia
}

\begin{abstract}
The problem of design engineering of anthropomorphic robot legs is considered. An overview of the existing anthropomorphic robots and an analysis of servomechanisms and bearing parts involved in the assembly of robot legs are presented. We propose an option for constructing the legs of the robot Antares under development. A two-motor layout, used in the knee, ensures higher joint power along with independent interaction with the neighboring upper and lower leg joints when bending. To reduce the electrical load on the main battery of the robot, the upper legs are provided with a mounting pad for additional batteries powering servos. Direct control of the servos is also carried out through the sub-controllers, responsible for all 6 engines installed in the articular joints of the robot legs.
\end{abstract}

\section{Introduction}

Among problems, which developers of mobile generalpurpose and special-purpose robots face nowadays, are issues of robot's flotation ability on the rugged terrain, autonomous movement and control of kinetic equipment [1]. A lever-hinge system of human and animals mobility, created by nature, is the most adapted to the natural earth's surface and is suitable for use during movement of the anthropomorphic robot [2].

Because of the lack of a unified methodology and software for engineering lever-hinge systems for anthropomorphic robots, developers are forced to create their own software in the design process of each individual robot [2]-[4].

The aim of this article is to analyze the existing solutions to the construction of lever-hinge mechanisms of the lower extremities (legs) of anthropomorphic robots and to develop the rational structure of legs of the robot Antares.

One of the simplest structures of the biped robot is described in [4]. It is made up of two-millimeter aluminum sheet, includes six servos operated by EyeBot controller, and weighs $1.11 \mathrm{~kg}$. When walking the robot reaches a speed of $120 \mathrm{~m} / \mathrm{h}$ at a maximum angle of 60 degrees between the hips. Such robot architecture with six servos is used in [5] to study the operating angles of the joints of the knee, ankle and hip.

The anthropomorphic robot in the HanSaRam series, which regularly participates in the FIRA league since 2000, is discussed in [6]. HanSaRam-VIII (HSR-VIII) robot has 28 servos, weighs $5.5 \mathrm{~kg}$ and can move at a speed of up to $12 \mathrm{~cm} / \mathrm{s}$.
In [7], the anthropomorphic robot Lola, having 7 degrees of freedom per leg, weighs $55 \mathrm{~kg}$ with the $180 \mathrm{~cm}$ height. The problems of the stability of the robot after stopping as well as a gradual contact of foot parts with the surface when walking are discussed. Elastic materials of the toe and heel of the robot foot ensure reduction of impact force on the robot structure during touching the surface.

For moving on complicated uneven surfaces, impassable for tracked or wheeled robots, more sophisticated non anthropomorphic structures are also being developed, with one [8], six [9], [10] and a large number [11] of lower extremities.

Based on the conducted analysis of structures of anthropomorphic robots, the robot Poppy of the French company INRIA Flowers and Darwin-OP robot of the company Trossen Robotics [12] were determined to be the closest analogues to the robot Antares under development. Let us consider these robots in more detail.

Modular robot design helps the researcher to change the movement of any robot's limb by isolating the desired limb from the rest of the body, almost without affecting the performance. The structure is specially designed for the installation of additional sensors and connection cables. In addition, such a design facilitates periodic robot maintenance service. However, the center of mass of Poppy is located in the solar plexus, which adversely affects load distribution. The robot becomes unstable and cannot move independently (only with the help of human).

Poppy has the same number of degrees of freedom as Antares in the pelvic region, distributing them in a different way, which makes it not so mobile in the knee and ankle joints. The leg joints have only 3 motors (one in each ankle, knee and hip joints). This adversely affects 
the overall mobility of the robot, judging by the video footage and design files of the robot available from the developers.

The robot Darwin-OP is a robot platform intended for research and development within the framework of educational process. DARwin OP has high performance and dynamic characteristics and a wide range of sensors. The robot communicates with people by using loudspeakers, microphones, cameras, tactile sensors, LEDs, hand gestures. It possesses 20 Dynamixel actuators that ensure free movement of limbs with a given accuracy and strength margin, as the gears are made of metal. The center of mass is located in the center of pelvis, which ensures the correct distribution of the load during walking and inertia, especially in the extremities. A modular robot design helps the researcher to change the movement of any limb of DARwin OP. The structure also allows the installation of additional sensors.

\section{Design of leg joint of the anthropomorphic robot antares}

Designing Antares included several steps associated with the development of joints of legs, arms, torso and head. First priority is given to designing the leg joint because of the following reasons: the high complexity of the layout of joint parts; necessity of this joint for robot movement in space; complexity of calculating joint unit due to the assumed highest load of parts relative to all other joints.

Total toe structure includes pelvic joint and two identical leg joints consisting of simpler attachment point to the pelvic, hip, knee, lower leg, ankle and foot units. Joints of robot pelvic and legs are developed in accordance with the proportions of the human body, adjusted for assumed growth. Their total length is 510.7 $\mathrm{mm}$. The length of ankle and hip joints is $20 \mathrm{~cm}$.

Since in the kinematics of the robot these joints act as a lever arm, this constructive solution is reasonable. The difference in length is achieved by connecting lower leg joint to the ankle joint which is, in turn, connected with the foot, while the upper leg joint is linked to the hip joint that is connected to the point of attachment to the hip unit.

Auxiliary batteries (an installation site is provided ) will be installed in the upper legs to reduce the electrical load on the main battery of Antares as well as to control actuator powering, which will help to avoid power supply problems. To save the processing power of the main controller and the computer located in the torso, auxiliary controllers will also be installed in the upper leg joints. These controllers are responsible for the work of all six engines of robotic leg joints. The given design complicates the calculation of the kinematics of the robot motion, but provides more complex movements.

Figure 1. A two-motor knee
Through the use of twin-engine layout in the knee (Fig. 1) a separate leg joint is obtained, which interacts with neighboring upper and lower leg joints, allowing them to be independent of each other when bending (Fig. 2). In addition, the use of two-motor knee simplifies the selection of servo, as in this type of knee the load is divided between two separate motors. Another advantage of this unit is that it facilitates design engineering of the above-mentioned joints of the upper and lower legs.

Interconnected plate lines, linked by screw couplings and cross plates, make up the basis of the leg structure of Antares (Fig. 3). For parts manufacturing, 2, 4 and 10 $\mathrm{mm}$ thick aluminum sheets were used. 6-mm-thick aluminum rods were applied for the crossties.

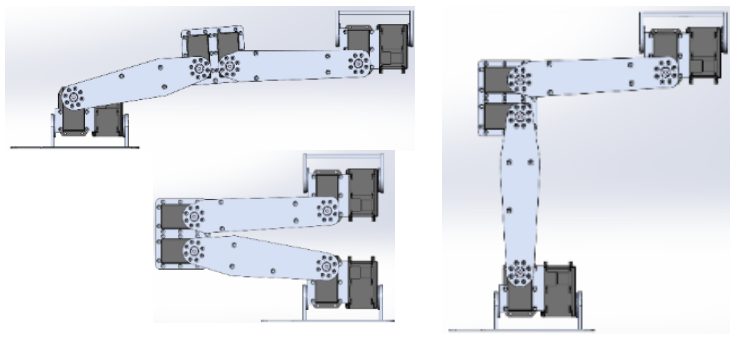

Figure 2. Functional capabilities of the two-motor knee

Parts made from $2 \mathrm{~mm}$ thick aluminum sheet are the basis of the joints of lower and upper legs, foot and pelvic attachment. The joints parts made from $2 \mathrm{~mm}$ sheet are structurally designed for power loads and pressure from top. Parts made from 4-mm-thick aluminum sheets are used as stiffening ribs intended for the torsional loads. From these sheets we made transverse upper and lower leg struts and mounting plates of the hip ( $4 \mathrm{~mm}$ thick) and foot. It is also intended to use them to locate the internal components, such as an auxiliary battery, actuators controller of the entire leg joint. Along with the transverse plates, aluminum bars are used as stiffening ribs but only as elements of structural reinforcement.

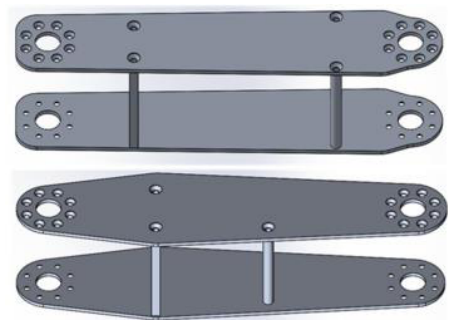

Figure 3. Upper and lower leg structures

In the tibial joint a broader transverse plate is used in order to achieve sufficiently reliable structure. This was necessary to ensure that the ankle could be used as efficiently as possible, which required the free use of the internal space of ankle joint. Screw coupling in this case is not only a stiffening rib, but also an arresting stop so that the ankle joint could not be broken and lead to the damage of other components while working. From 10 mm thick aluminum sheets one type of parts is made - a special bearing plate used for the assembly of hip and ankle joints.

For motors Dynamixel MX-64, used in the leg joints, the flanges were made that are necessary for linking 
separate joints parts and components to provide mobility and stability. The flanges are located in a special socket on the motor housing and the bearing and secured by a special cover which prevents the collapse of the structure during motion and because of vibration. An obligatory requirement for the bearing is a height of $3.5 \mathrm{~mm}$, to comply with the centering of axial arrangement of engines in the overall structure, which is important during robot movement.

Pelvic mechanism is located in the lower part of the torso of Antares and is designed for the axial legs rotations as well as for accommodating the main battery. The construction includes two bushings, gears with gear ratio 1: 1, two motors Dynamixel MX-28, two 2- mmthick plates, and the flange bearings $8 \times 14$ (Fig. 4). The choice of less powerful servos, compared with those used in the construction of the other legs joints, is substantiated by the fact that for axial rotations the high power of engines is not required.

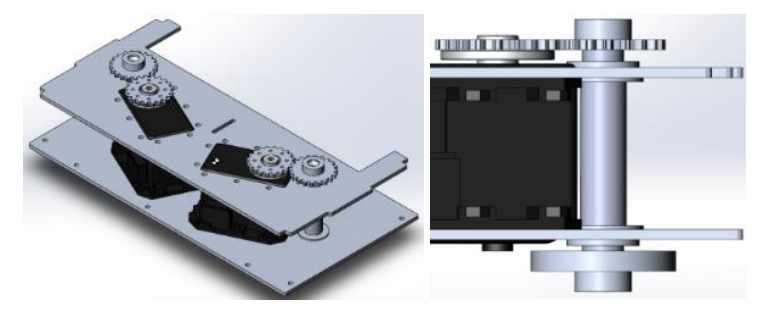

Figure 4. Pelvic mechanism

The hip and ankle joints are formed by pairwise motor connection with metal inserts; in the motor housing extra strong plastic or aluminum are used in order to withstand physical loads on the motor housing during movement (Fig. 5).

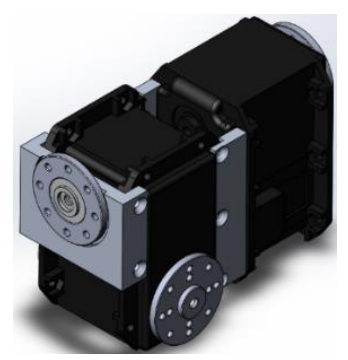

a)

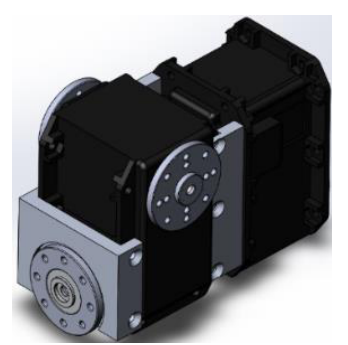

b)
Figure 5. Joint assemblies of: a) hip; b) ankle

Pairwise motor connection provides space saving in the construction, in order to avoid excessive massiveness. This is necessary to ensure the mobility and flexibility of the assembled joints. In addition, the construction of the hip and ankle joints is designed to the highest possible reproduction of functionality of human ankle and hip joints which are similar to a spherical joint with a limited angle of rotation. Thus, to reproduce the human joint structure, it was decided to introduce to these joints two cylindrical hinges with mutually perpendicular axes shown in Fig. 5.

This solution is applied because, at the moment, it is not possible to repeat structure of the spherical joint and make it controllable to the full for the anthropomorphic mechanism, sufficiently reliable and compact, relatively inexpensive and not requiring constant maintenance. Achieving all of this will complicate both the construction itself and the control of the robot. The implemented solution, in turn, simplifies the structure of the robot ankle without compromising ankle capabilities.

\section{Experimental results and discussion}

The leg construction provides large steering angles for the motors, which ensures greater flexibility and ductility of the joint compared with the mentioned analogs. The robot can easily do the splits, raise the leg straight or at an angle, bend it at the knee, while keeping the foot parallel to the floor, if necessary. There are a variety of sit-ups rather than some specific predetermined movements seen in the analogues, including touching the floor by the pelvic mechanism without the threat of damaging its joints. Pelvic mechanism provides axial rotation of the robot lower limbs, which has a positive effect on its portability and allows it to rotate on the way with a minimum turning radius, turn in the necessary direction on the spot and perform a wider range of different leg movements than those available to humans.

Pelvic mechanics allows the robot to rotate each leg at $360^{\circ}$ around its axis; however, at this stage we limited it up to $270^{\circ}$, since there was no need for such a large range of rotation. The angle of flexion at the hip joint is $120^{\circ}$. The maximum extension angle is $45^{\circ}$. The side lunge of the leg is $+90^{\circ}$. Similar to humans, it is impossible to fully bring the leg back, as the lower limbs will touch each other. The maximum bringing back of the leg is $55^{\circ}$. The legs are brought back up to the moment when they almost touch each other, and this limits the range of the stroke. The side lunge of the leg is limited to an angle of $135^{\circ}$. It was decided to set limits up to $90^{\circ}$. This is due to the fact that the robot should resemble the structure of the human, and this angle allows the robot to do the splits. In this case, there is no need to have a greater range of variations of the leg position in the hip joint. The knee joint comprises two servos combined into one unit. This design solution increases the mobility and strength torque of the knee. This allows the actuator to bend the knee at an angle of $162^{\circ}$. The use of two actuators in the knee joint entails constant synchronization of the motors relative to each other, since one actuator does not provide the same large angle of the displacement in a joint without a significant loss in power and mobility of the structure. The result is that the location of motors corresponds to the perpendicular position relative to the frontal plane of the robot. The ankle design provides the eversion - the displacement of the foot inwards to the sagittal plane as well as the inversion - shifting of the foot outwards from the sagittal plane. Changing the generalized coordinate of the ankle joint in roll represents a rotation of the foot relative to the upper limb from the neutral position. The angle of the foot position changes: eversion is $90^{\circ}$ and inversion is $90^{\circ}$. Moving toe upwards relative to the neutral position is taken as a positive angle, and downwards - as the negative angle. In this case, the pitch angle of the foot deviates from $+88^{\circ}$ to $-180^{\circ}$. Fig. 
6 shows the results of work to change the generalized coordinate of the robot foot. Movement is carried out in full compliance with design feature that limits the angles of pitch and roll.
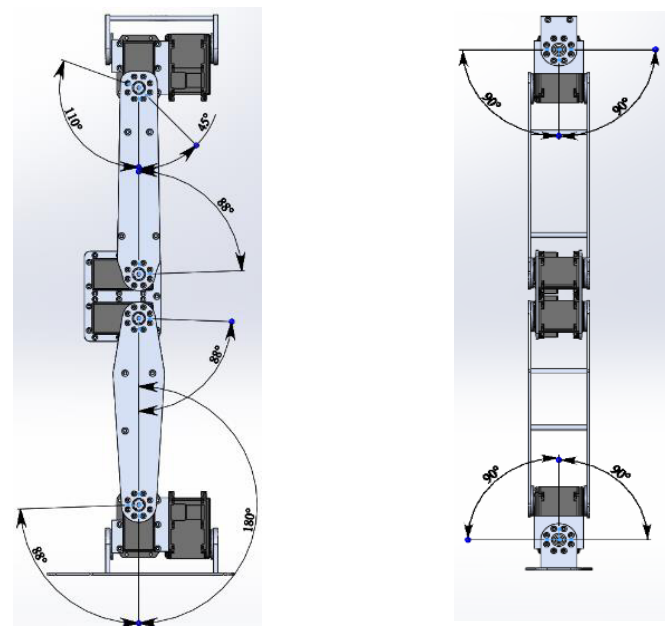

Figure 6. Turning angles of the leg

Apart from the pelvic mechanism responsible for axial rotations of legs and being a separate unit, in the legs structure we applied actuators Dynamixel MX-64. Each Dynamixel has a unique ID for connection to the common data line, supports the connection TTL, RS485 and others, can be connected to the common control bus; available LED or emergency shutdown (torque-off) functions may be set to predetermined values of temperature, current and voltage. These actuators can be adjusted to move more smoothly. Dynamixel servos can be controlled from a PC or a microcontroller, which is a great advantage in the development of prototypes. Without the battery and the controller installed in the hip joint, the estimated total weight of the metal frame, servos and flange connections is $1.07 \mathrm{~kg}$, of which $756 \mathrm{~g}$ account for the Dynamixel MX-64 servos. Construction of the two joints of the leg and pelvic mechanism weighs $2.44 \mathrm{~kg}$.

Table 1. The comparison of the angles of the human and the robot Antares

\begin{tabular}{|l|l|l|}
\hline & \multicolumn{1}{|c|}{ Human } & \multicolumn{1}{c|}{ Antares } \\
\hline Range by the pitch of foot & $\begin{array}{l}\text { from }-50^{\circ} \text { to } \\
40^{\circ}\end{array}$ & $\begin{array}{l}\text { from }-88^{\circ} \text { to } \\
180^{\circ}\end{array}$ \\
\hline Range by the roll of foot & $\begin{array}{l}\text { from }-30^{\circ} \text { to } \\
20^{\circ}\end{array}$ & $\begin{array}{l}\text { from }-90^{\circ} \text { to } \\
90^{\circ}\end{array}$ \\
\hline $\begin{array}{l}\text { Range of flexion-extension, } \\
\text { for the hip }\end{array}$ & $\begin{array}{l}\text { from }+120^{\circ} \text { to } \\
20^{\circ}\end{array}$ & $\begin{array}{l}\text { from }+110^{\circ} \\
\text { to } 45^{\circ}\end{array}$ \\
\hline $\begin{array}{l}\text { Range of flexion-extension, } \\
\text { for the knee }\end{array}$ & $\begin{array}{l}\text { from } 0^{\circ} \text { to } \\
110^{\circ}\end{array}$ & $\begin{array}{l}\text { from } 0^{\circ} \text { to } \\
162^{\circ}\end{array}$ \\
\hline $\begin{array}{l}\text { Range of the side lunge of } \\
\text { the leg in the hip }\end{array}$ & from $0^{\circ}$ to $45^{\circ}$ & $\begin{array}{l}\text { from } 0^{\circ} \text { to } \\
90^{\circ}\end{array}$ \\
\hline $\begin{array}{l}\text { Range of the relative } \\
\text { bringing back of the leg in } \\
\text { the hip }\end{array}$ & from $0^{\circ}$ to $30^{\circ}$ & $\begin{array}{l}\text { from } 0^{\circ} \text { to } \\
55^{\circ}\end{array}$ \\
\hline Range of rotation for the hip & $\begin{array}{l}\text { from }-45^{\circ} \text { to } \\
45^{\circ}\end{array}$ & $\begin{array}{l}\text { from }-45^{\circ} \text { to } \\
45^{\circ}\end{array}$ \\
\hline
\end{tabular}

Table 1 shows a comparison of the different angles of deflection of legs positions of the human and developed prototype of an anthropomorphic robot Antares. Based on this table, we can conclude that the various actions typical of the human and the robot can be executed by the latter, as the ranges of changes of human positions angles lie within the ranges of changes of robot angles.

It should be noted that, except for the range of the foot roll, all other angles are artificially limited in order to avoid unnecessary contact of the parts lying in a single plane at the time of motion. Since the robot represents a robotic platform intended for scientific research and development within the framework of educational process, its modular nature can help the researcher to change the movement of any limb by isolating the desired limb from the rest of the body, almost without affecting performance. The structure is specially designed for the installation of additional sensors and connection cables. In addition, such a design facilitates periodic robot maintenance service.

Load weight balancing in the legs of the robot taking into account the maximum supposed weight of $8 \mathrm{~kg}$ occurs according to the following formula:

$$
F=P / 2=80 / 2=40 \mathrm{~N}
$$

where, $\mathrm{F}$ is the force applied to the lower leg assembly; $\mathrm{P}$ is a weight, which keeps the lower leg; the denominator -2 , since all the weight is distributed over 2 legs. Fig. 7 shows the torque supplied to the motor shaft of the ankle in the process of raising the legs. The graph shows that the ankle motor has a maximum torque when the robot is in a sitting position. The tension in the construction of the lower and upper leg with a total weight of the robot equal to 8 kilograms is $7.44 * 10^{\wedge} 5 \mathrm{~N} / \mathrm{m}^{\wedge} 2$.

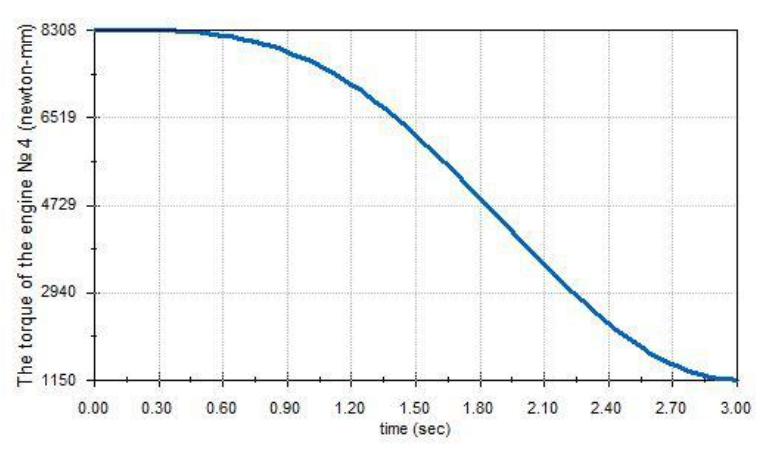

Figure 7. Torque supplied to the motor shaft

The conducted study on the maximum load revealed that the lower leg can withstand a load of 1040 Newtons. Summarizing, we can say that the lower and upper leg individually can withstand 13 -fold vertical load on the structure, without succumbing to deformation and breakage. It was taken into consideration that fasteners for flange connections were fixed and immobile.

This research devoted to mechanical engineering of leg joints of anthropomorphic robot is focus on development of technical means and mobile robots, in order to modeling collaborative work of users, robots and distributed means of cyberphysical environment [13]-[17]. 


\section{Conclusion}

The conducted analysis showed the presence of anthropomorphic robot models from $30 \mathrm{~cm}$ to $180 \mathrm{~cm}$ high with a different number of degrees of freedom and kinematic schemes. It was concluded that the closest analogues to the robot Antares under development are Poppy and Darwin-OP robots. A two-motor layout, used in the knee, ensures higher joint power along with independent interaction with the neighboring upper and lower leg joints when bending. The electrical load on the main battery of Antares is reduced by the use of auxiliary batteries installed in the upper legs and powering the servos. The direct servo control is also performed by the auxiliary controllers responsible for the work of all six engines of the leg joints. Studies of the prototype design have demonstrated that individual components and parts have a more than ten-fold safety margin. The developed robot is intended for creation of assistive technologies via human-robot interaction based on multimodal interfaces in cyberphysical intelligent environment.

The study was performed through the grant of the Russian Science Foundation (project no. 16-19-00044).

\section{References}

1. V.B. Kudryashov, V.S. Lapshov, V.P. Noskov, I.V. Rubtsov. Problems of robotization for military ground technics, Izvestiya SFedU. Engineering Sciences, 3/152: 42-57, (2014).

2. A.K. Kovalchuk, D.B. Kulakov, S.E. Semenov, V.V. Yarots, A.A. Vereikin, B.B. Kulakov, L.A. Karginov. Method for designing spatial tree-like actuators of walking robots, Engineering bulletin of the Bauman MSTU, 11: 6-10, (2014).

3. V.V. Lapshin. About the walking motion stability, Science and Education of the Bauman MTSU, 6: 319-335, (2014).

4. S. Warnakulasooriyaa, A. Bagheria, N. Sherburnb, M. Shanmugavel. Bipedal Walking Robot - A developmental design, Procedia engineering, 41: 1016-1021, (2012).

5. S.C. Lima, G.H. Yeapa. The Locomotion of Bipedal Walking Robot with Six Degree of Freedom, Procedia Engineering, 41: 8-14, (2012).
6. Yoo J.K., Lee B.J., Kim. J.H. Recent Progress and Development of the Humanoid Robot Hansaram, Robotics and Autonomous Systems, 57: 973-981, (2009).

7. T. Buschmann, S. Lohmeier, H. Ulbrich. Humanoid Robot Lola: Design and Walking Control // Journal of Physiology, 103: 141-148, (2009).

8. X. Yua, C. Fub, K. Chen. Modeling and Control of a Single-legged Robot, Procedia Engineering, 24: 788792, (2011).

9. H. Rostro-Gonzalez, P.A. Cerna-Garcia, G. TrejoCaballero, C.H. Garcia-Capulin, M.A. IbarraManzano, J.G. Avina-Cervantes, C. Torres-Huitzil. A CPG System Based on Spiking Neurons for Hexapod Robot Locomotion, Neurocomputing, 170: 47-54, (2015).

10. P.S. Pan, C.M. Wu. Design of a Hexapod Robot with a Servo Control and a Man-Machine Interface, Robotics and Computer-Integrated manufacturing, 28: 351-358, (2012).

11. R. Vidoni, A. Gasparetto. Efficient Force Distribution and Leg Posture for a Bio-Inspired Spider Robot, Robotics and Autonomous Systems, 59: 142-150, (2011).

12. «ROBOTIS» catalogue. http://en.robotis.com/index/product.php?cate_code $=1$ 01011

13. A.A. Karpov, A. Lale, A.L. Ronzhin. Multimodal assistive systems for a smart living environment. SPIIRAS Proceedings, 19: 48-64, (2011).

14. A.I. Motienko, A.L. Ronzhin, N.A. Pavlyuk. Modern developments of rescue robots: possibilities and principles of their application, Science Bulletin of the NSTU, 3/60: 147-165, (2015).

15. A.L. Ronzhin, V.Yu. Budkov. Multimodal Interaction with Intelligent Meeting Room Facilities from Inside and Outside, NEW2AN/ruSMART 2009, LNCS 5764: 77-88, (2009).

16. R.M. Yusupov, A.L. Ronzhin. From Smart Devices to Smart Space, Herald of the Russian Academy of Sciences, 80/1: 45-51, (2010).

17. A.A. Karpov, A.L. Ronzhin. Information Enquiry Kiosk with Multimodal User Interface, Pattern Recognition and Image Analysis, 19/3 (Moscow: MAIK Nauka/Interperiodica): 546-558, (2009). 